ELECTRONIC RESEARCH ANNOUNCEMENTS

OF THE AMERICAN MATHEMATICAL SOCIETY

Volume 13, Pages 46-52 (May 14, 2007)

S $1079-6762(07) 00173-4$

\title{
GLOBAL WEAK SOLUTIONS OF NON-ISOTHERMAL FRONT PROPAGATION PROBLEM
}

\author{
BO SU AND MARTIN BURGER
}

(Communicated by Luis A. Caffarelli)

\begin{abstract}
We show the global existence of weak solutions for a free-boundary problem arising in the non-isothermal crystallization of polymers. In particular, the free interface is shown to be of codimension one for every time $t$ in two space dimensions; Hölder continuity of the temperature $u$ is proven.
\end{abstract}

\section{InTRODUCTION AND MAIN RESULTS}

We are interested in the global existence of weak solution to the following system of partial differential equations, which is the level-set formulation of the nonisothermal crystallization process of polymers (see [5, 20]):

$$
\begin{gathered}
\partial_{t}(u+\chi(\varphi))-\Delta u=0, \\
\varphi_{t}+G(u)|\nabla \varphi|=0, \\
u(x, 0)=u_{0}(x), \quad \varphi(x, 0)=\varphi_{0}(x),
\end{gathered}
$$

where

$$
\chi(\varphi)=\operatorname{sign}(\varphi)= \begin{cases}-1 & \text { if } \varphi<0 \\ {[-1,1]} & \text { if } \varphi=0 \\ 1 & \text { if } \varphi>0\end{cases}
$$

Based upon the physics of the problem, we assume throughout the paper that for all $u, u_{1}, u_{2} \in \mathbb{R}$,

$$
\begin{aligned}
& 0<C_{1} \leq G(u) \leq C_{2}<\infty, \\
& \left|G\left(u_{1}\right)-G\left(u_{2}\right)\right| \leq L\left|u_{1}-u_{2}\right| .
\end{aligned}
$$

In the isothermal case - the Stefan problem - the temperature is fixed on the moving interface. Therefore, the weak formulation of the classical Stefan problem is as follows:

$$
\partial_{t}(u+\chi(u))-\Delta u=0 .
$$

There is vast literature on the study of Stefan problems (see, e.g., 22, 19, 24, 36).

Received by the editors September 15, 2006.

2000 Mathematics Subject Classification. Primary 70H20, 35R35, 35L45.

Key words and phrases. Free boundary, level-set method, heat conduction, growth, crystallization, Hausdorff measure, codimension-one-measure estimate, decomposition. 
However, the best known regularity of the temperature $u$ for (1.7) is just continuity (see [8, 14, 37, 30]), and the codimension-one property of the moving front is still open.

Because of the change of temperature on the interface in the non-isothermal polymer crystallization, $\operatorname{sign}(u)$ in the Stefan problem (1.7) is replaced by $\operatorname{sign}(\varphi)$ in (1.1), where $\varphi$ is governed by the Hamilton-Jacobi equation (1.2). Earlier works by Avrami [1, Kolmogorov [21, and Evans [17] in the 1930s and 1940s addressed spatially homogeneous growth of crystals, where the phase could be computed analytically.

Recently the modeling of non-isothermal crystallization has been extensively studied in 27, 31, 34, 29, 15, 16, 3, 5, 6, 77. The local existence of a single smooth moving front in (1.1)-(1.3) was shown under the assumption that initial interface is a small perturbation of a sphere in Friedman-Velazquez [20. In practice there are many crystals created by nucleation so that a situation with multiple crystals should be studied instead. As crystallines grow, they definitely infringe each other in finite time as long as there is a positive lower bound on the propagation speed. Consequently, the moving front may not be smooth.

To capture the physical features of front propagation, we study a global weak solution of (1.1)-(1.3). In this paper, we prove the global existence of a weak solution of (1.1) - (1.3) for large initial data. In particular, we show that temperature is Hölder continuous in space and the moving front is a Lipschitz graph in timespace.

The main novel features of our work are:

(i) the moving interface is shown to be of codimension one;

(ii) finite Hölder continuous propagation speed yields an intrinsic estimate of finite codimension-one Hausdorff measure of the moving interface for every time $t$ in two space dimensions;

(iii) based upon (ii), we prove Hölder continuity of the temperature $u$ by a decomposition argument.

Now we need to interpret the weak solution $(\varphi, u)$ of (1.1) and (1.2). The temperature $u$ satisfies (1.1) in the standard distributional sense. The best available Hölder regularity of the temperature $u$ is weaker than Lipschitz continuity due to the non-smoothness of the moving interface, and so is the normal velocity of the moving interface. In reality, there is also thermal noise in the heat transfer. Then the temperature $u$ may at best be Hölder continuous, and consequently the phase function $\varphi$ has to be understood in an $L^{\infty}$ sense (see [10, 11, 12]) since the level-set equation (1.2) allows a discontinuity to develop in finite time even if the initial phase function is smooth (because of the non-uniqueness of ODE solutions given a Hölder continuous right-hand side).

In this paper, we shall use the notion of $L^{\infty}$ solution of Hamilton-Jacobi equations.

Denote $\{\varphi(\cdot, t)<0\}$ by $\Omega(t)$ and let $\Gamma(t)=\partial \Omega(t)$. Our main theorem is stated as follows.

Theorem 1.1. Assume that the initial front $\Gamma(0)$ is $\bigcup_{i=1}^{m} \Gamma_{i}^{0}$, where each $\Gamma_{i}^{0}$ is a curve satisfying the local flattening condition: for any points $A$ and $B$ on $\Gamma_{i}^{0}$ with $L(\overline{A B})<1, \sup _{x \in \widetilde{A B}} \operatorname{dist}(x, \overline{A B}) \leq \xi L(\overline{A B})^{1+\beta}$, where $\widetilde{A B}$ is the connecting part of $\Gamma_{i}^{0}$ between the point $A$ and $B$. Moreover, let $\left\|u_{0}\right\|_{C^{0,1}\left(\mathbb{R}^{2}\right)} \leq C$. Then for any 
$T>0$, the temperature $u$ of (1.1) - (1.3) satisfies

$$
\begin{gathered}
\|u\|_{L^{\infty}\left(\mathbb{R}^{2} \times[0, T]\right)} \leq C, \\
\left\|u\left(\cdot, t_{1}\right)-u\left(\cdot, t_{2}\right)\right\|_{L^{p}\left(\mathbb{R}^{2} \times[0, T]\right)} \leq C\left|t_{1}-t_{2}\right|^{\gamma} \text { for any } \gamma \in\left(0, \frac{1}{2}\right), \\
\left|u\left(x_{1}, t\right)-u\left(x_{2}, t\right)\right| \leq C(\alpha)\left|x_{1}-x_{2}\right|^{\alpha} \text { for any } \alpha \in(0,1),
\end{gathered}
$$

for any $x_{1}, x_{2} \in \mathbb{R}^{2}$ and $t_{1}, t_{2}>0$. The phase function $\varphi \in L^{\infty}\left(R^{2} \times(0, T]\right) \cap$ $B V_{l o c}\left(R^{2} \times(0, T]\right)$ satisfies $\partial\{\varphi<0\}=\partial\{\varphi>0\}$. Furthermore, $\partial\{\varphi<0\}$ is a Lipschitz graph in time-space, i.e., $\left|\gamma\left(y_{1}, \varphi\right)-\gamma\left(y_{2}, \varphi\right)\right| \leq \frac{\left|y_{1}-y_{2}\right|}{C_{1}}$. For each time $t$, $\Gamma(t)$ satisfies

$$
\mathcal{H}^{1}\left(\Gamma(t) \cap B_{r}\left(y_{0}\right)\right) \leq C\left(m, \xi, \beta, \alpha, C_{1}, C_{2}, r\right),
$$

for any $y_{0} \in \mathbb{R}^{2}$. As $r \rightarrow 0, \mathcal{H}^{1}\left(\Gamma(t) \cap B_{r}\left(y_{0}\right)\right) \rightarrow 0$ for each $t \in(0, T]$.

\section{OutLine OF PROOF}

To study (1.1)-(1.3), we consider the following approximate equations:

$$
\begin{aligned}
& \frac{\partial}{\partial t}\left(u+\chi_{\epsilon}(x, t, \varphi)\right)-\Delta u=0 \quad \text { in } R^{n} \times[0, T], \\
& \frac{\partial \varphi}{\partial t}+G(u)|\nabla \varphi|=0 \quad \text { in } R^{n} \times[0, T], \\
& u(x, 0)=u_{0}(x), \quad \varphi(x, 0)=\varphi_{0}(x) .
\end{aligned}
$$

Here $\chi_{\epsilon}$ is defined by

$$
\chi_{\epsilon}(x, t, \varphi)= \begin{cases}-1 & \text { if } t-\gamma(x, \varphi)>2 \epsilon, \\ -1+\frac{\gamma(x, \varphi)-t}{\epsilon} & \text { if } 0 \leq t-\gamma(x, \varphi) \leq 2 \epsilon, \\ 1 & \text { if } \varphi(x, t)>0,\end{cases}
$$

where $\gamma(x, \varphi)=\inf \{s: \varphi(x, s)<0\}$ for a monotone decreasing function $\varphi$ in time $t$.

Remark 2.1. Observe that the solution of the level-set equation (2.2) is monotone decreasing in time; thus $\gamma$ is well defined.

We introduce the following notation. Suppose that $A \subset R^{n}$ and $B \subset R^{n}$; then the distance between the sets $A$ and $B$ is given by

$$
\operatorname{dist}(A, B)=\sup _{x \in A} \operatorname{dist}(x, B)+\sup _{y \in B} \operatorname{dist}(y, A) .
$$

Let $T$ be any given positive number. Denote by $\Gamma_{\phi}^{-}$the boundary of $\{\phi<0\}$ in $R^{n} \times(0, T]$, and by $\Gamma_{\phi}^{+}$the boundary of $\{\phi>0\}$ in $R^{n} \times(0, T]$ for a function $\phi$ monotone decreasing in time $t$. We need the following assumption on the initial front before we state the approximation lemma:

$$
m\left(\partial\left\{\varphi_{0}<0\right\} \cap \partial\left\{\varphi_{0}>0\right\} \cap\left\{\varphi_{0}=0\right\}\right)=0 .
$$

We denote $\left\{\varphi_{0}<0\right\}$ by $\Gamma(0)$ as long as the assumption (A1) holds.

Lemma 2.2. Suppose that the initial temperature satisfies $u_{0} \in C^{2}\left(R^{n}\right) \cap L^{\infty}\left(R^{n}\right)$, and the initial almost-everywhere-continuous phase function $\varphi_{0} \in L^{\infty}\left(R^{n}\right)$ satisfies the assumption (A1) with the following property: $\varphi_{0}<-1$ if $\varphi_{0} \leq 0$, and $\varphi_{0}>1$ if $\varphi_{0}>0$. The material function $G$ satisfies assumptions (1.5) and (1.6). Then for every $\epsilon>0$, there exists a solution $u^{\epsilon} \in W_{x, t}^{2,1, p}\left(R^{n} \times(0, T]\right) \cap C^{\frac{\alpha}{2}, \alpha}\left(R^{n}\right)$ to 
(2.1) - (2.3) with $\partial\left\{\varphi^{\epsilon}<0\right\}=\partial\left\{\varphi^{\epsilon}>0\right\}$, for any $p \in(1, \infty)$ and $\varphi^{\epsilon} \in L^{\infty}\left(R^{n} \times\right.$ $(0, T]) \cap B V\left(R^{n} \times(0, T]\right)$. Furthermore, $\partial\left\{\varphi^{\epsilon}<0\right\}$ is a Lipschitz graph in time-space independent of $\epsilon$, i.e., $\left|\gamma\left(y_{1}, \varphi^{\epsilon}\right)-\gamma\left(y_{2}, \varphi^{\epsilon}\right)\right| \leq \frac{\left|y_{1}-y_{2}\right|}{C_{1}}$.

Then we establish a surprising theorem in two spatial dimensions that the onedimensional Hausdorff measure of the moving front is finite if the normal velocity $f(x, t)$ is Hölder continuous with respect to $x$ and is bounded from below. In other words, there is a discontinuous $L^{\infty}$ solution $\varphi$ of the following level-set equation, whose level set $\partial\{\varphi<0\}=\partial\{\varphi>0\}$ is of finite codimension-one Hausdorff measure for every time $t>0$ :

$$
\begin{aligned}
& \varphi_{t}+f(x, t)|D \varphi|=0, \quad x \in \mathbb{R}^{2}, t>0, \\
& \varphi(x, 0)=\phi(x),
\end{aligned}
$$

where $0<C_{1} \leq f(x, t) \leq C_{2}$.

Theorem 2.3. Let the moving front $\Gamma(t)$ be the boundary of the reachable set $\Omega(t)$ driven by the normal velocity $f(x, t)$, which is Hölder continuous with $\mid f\left(x_{1}, t\right)-$ $f\left(x_{2}, t\right)|\leq \kappa| x_{1}-\left.x_{2}\right|^{\alpha}$ and $0<C_{1} \leq f(x, t) \leq C_{2}$. Assume that the initial front $\Gamma(0)$ is $\bigcup_{i=1}^{m} \Gamma_{i}^{0}$, where $\Gamma_{i}^{0}$ is the curve satisfying the local flattening condition: for any points $A$ and $B$ on $\Gamma_{i}^{0}$ with $L(\overline{A B})<1, \sup _{x \in \widetilde{A B}} \operatorname{dist}(x, \overline{A B}) \leq \xi L(\overline{A B})^{1+\beta}$, where $\widetilde{A B}$ is the connecting part of $\Gamma_{i}^{0}$ between the points $A$ and $B$. Then for any $t>0, \Gamma(t)$ satisfies

$$
\begin{gathered}
\mathcal{H}^{1}\left(\Gamma(t) \cap B_{r}\left(y_{0}\right)\right) \leq C\left(m, \xi, \beta, \alpha, C_{1}, C_{2}\right) r \quad \text { if } r \in\left(0, \kappa^{\frac{-2+\alpha^{2}}{\alpha}}\right], \\
\mathcal{H}^{1}\left(\Gamma(t) \cap B_{r}\left(y_{0}\right)\right) \leq C\left(m, \xi, \beta, \alpha, C_{1}, C_{2}\right) r^{2} \kappa^{\frac{2-\alpha^{2}}{\alpha}} \text { if } r \geq \kappa^{\frac{-2+\alpha^{2}}{\alpha}},
\end{gathered}
$$

for any $y_{0} \in \mathbb{R}^{2}$.

The argument is quite lengthy due to geometric complexity. Roughly speaking, in the first step, we show that the "convex" part of the moving front is bounded by the lower boundary of the convex hull of the curve; we show that the complement of the "convex" part, which consists of countably many "vaguely concave" parts, is locally nicely behaved; that is, each "vaguely concave" part is close to a straight line in the sense that the "concavity" is small. Then we decompose each "vaguely concave" part into no more than three subparts; the union of the lower boundaries of the convex hulls of the subparts serves as the first approximation of the moving front. We iterate the argument to treat each "vaguely concave" subpart to produce the $n$th approximation of the moving front. As the iteration number of the above procedure goes to infinity, the moving front becomes locally flat, and thus completely restored as a 1-dimensional curve, and its length is finite.

For the purpose of an a priori regularity estimate of temperature, we must weaken the assumption on the Lipschitz constant. From now on, we assume that the velocity function $f(x, t)$ satisfies

$$
\left|f\left(x_{1}, t\right)-f\left(x_{2}, t\right)\right| \leq \kappa(t)\left|x_{1}-x_{2}\right|^{\alpha}, \text { where } \kappa \in L^{p}(0, T),
$$

Denote by $\mathcal{K}=\|\kappa\|_{L^{p}(0, T)}$ for $p>1$.

Theorem 2.4. Let the moving front $\Gamma(t)$ be the boundary of the reachable set $\Omega(t)$ driven by the normal velocity $f(x, t)$, which is Hölder continuous in space with $\left|f\left(x_{1}, t\right)-f\left(x_{2}, t\right)\right| \leq \kappa(t)\left|x_{1}-x_{2}\right|^{\alpha}, 0<C_{1} \leq f(x, t) \leq C_{2}$, and $\kappa \in L^{p}(0, T)$ for sufficiently large $p>1$. Denote $\|\kappa\|_{L^{p}(0, T)}$ by $\mathcal{K}$. Assume that the initial front $\Gamma(0)$ 
is $\bigcup_{i=1}^{m} \Gamma_{i}^{0}$, where $\Gamma_{i}^{0}$ is the curve satisfying the local flattening condition: for any points $A$ and $B$ on $\Gamma_{i}^{0}$ with $L(\overline{A B})<1$, $\sup _{x \in \widetilde{A B}} \operatorname{dist}(x, \overline{A B}) \leq \xi L(\overline{A B})^{1+\beta}$, where $\widetilde{A B}$ is the connecting part of $\Gamma_{i}^{0}$ between the point $A$ and $B$. Then for any $t>0$ and any $y_{0} \in \mathbb{R}^{2}, \Gamma(t)$ satisfies

$$
\begin{gathered}
\mathcal{H}^{1}\left(\Gamma(t) \cap B_{r}\left(y_{0}\right)\right) \leq C\left(m, \xi, \beta, \alpha, C_{1}, C_{2}\right) r \quad \text { if } r \in\left(0, \mathcal{K}^{\frac{-2+\alpha^{2}}{\alpha\left(1+\frac{1}{p}\right)-\frac{1}{p}}}\right] ; \\
\mathcal{H}^{1}\left(\Gamma(t) \cap B_{r}\left(y_{0}\right)\right) \leq C\left(m, \xi, \beta, \alpha, C_{1}, C_{2}\right) r^{2} \mathcal{K}^{\frac{2-\alpha^{2}}{\alpha\left(1+\frac{1}{p}\right)-\frac{1}{p}}} \quad \text { if } r \geq \mathcal{K}^{\frac{-2+\alpha^{2}}{\alpha\left(1+\frac{1}{p}\right)-\frac{1}{p}}} .
\end{gathered}
$$

Based upon Theorem 2.4 and Lemma 2.2, using a solution formula, we employ a decomposition argument to derive the desired Hölder estimate for $u^{\epsilon}(x, t)$ and $\nabla u^{\epsilon}(x, t)$.

Theorem 2.5. Assume that the initial front $\Gamma(0)$ is $\bigcup_{i=1}^{m} \Gamma_{i}^{0}$, where $\Gamma_{i}^{0}$ is the curve satisfying the local flattening condition: for any points $A$ and $B$ on $\Gamma_{i}^{0}$ with $L(\overline{A B})<1, \sup _{x \in \overparen{A B}} \operatorname{dist}(x, \overline{A B}) \leq \xi L(\overline{A B})^{1+\beta}$, where $\widetilde{A B}$ is the connecting part of $\Gamma_{i}^{0}$ between the point $A$ and $B$. Let $\left\|u_{0}^{\epsilon}\right\|_{C^{0,1}\left(\mathbb{R}^{2}\right)} \leq C$. Then the temperature $u^{\epsilon}$ of (2.1) -(2.3) enjoys

$$
\begin{gathered}
\left\|u^{\epsilon}\right\|_{L^{\infty}\left(\mathbb{R}^{2} \times[0, T]\right)} \leq C, \\
\left\|u^{\epsilon}\left(\cdot, t_{1}\right)-u^{\epsilon}\left(\cdot, t_{2}\right)\right\|_{L^{p}\left(\mathbb{R}^{2} \times[0, T]\right)} \leq C\left|t_{1}-t_{2}\right|^{\beta} \text { for any } \beta \in\left(0, \frac{1}{2}\right), \\
\left|u^{\epsilon}\left(x_{1}, t\right)-u^{\epsilon}\left(x_{2}, t\right)\right| \leq C(\alpha)\left|x_{1}-x_{2}\right|^{\alpha} \text { for any } \alpha \in(0,1),
\end{gathered}
$$

for any $x_{1}, x_{2} \in \mathbb{R}^{2}$ and $t_{1}, t_{2}>0$.

Because of finite propagation speed of the moving front, the decomposition is conducted in such a way that the contribution from the parts away from the point $(x, t)$ in the large scale is bounded by a coarse estimate of the moving front based upon Lemma 2.2, and that of the parts close to $(x, t)$ in the small scale is estimated with the aid of Theorem 2.4. In our argument, the finite speed of propagation of the moving front plays an essential role.

It follows from Theorem 2.5, Lemma 2.2, the $B V_{l o c}$ estimate for the level-set function $\varphi^{\epsilon}$ (see 12, 32]), and the definition of $L^{\infty}$ solution $\varphi^{\epsilon}$ of the HamiltonJacobi equation that the a priori $C^{0, \alpha}$ estimate in space of the temperature $u^{\epsilon}$, the $C^{0, \frac{1}{3}}$ estimate in time of $u^{\epsilon}$, and the uniform $B V_{l o c}$ estimate of $\varphi^{\epsilon}$ ensure that the approximate solution $\left(u^{\epsilon}, \varphi^{\epsilon}\right)$ of (2.1) -(2.3) converges to the solution $(u, \varphi)$ of (1.1)-(1.3) as $\epsilon \rightarrow 0$. Thus we conclude our main Theorem 1.1.

\section{Discussions}

In this paper we have developed a new concept of weak solutions for a nonisothermal phase-change model of polymeric materials, which allows us, in particular, to treat the growth and impingement of multiple crystals in a sound mathematical way. As a major ingredient of an existence proof we have derived results on the geometric properties of the front and the Hölder continuity of the temperature.

The important physical question is the stability of the moving front. In the manufacturing process, the interface between crystal (solid phase) and melt (liquid phase) should be well controlled so that high-quality polymers can be produced. The lately discovered scaling law of crystallization of polymers is different from that of metal solidification (see [35]). The study of the moving front for metal 
solidification was extensive, though a rigorous mathematical justification is completely open. We will address the stability of the non-isothermal moving front for polymerical materials in the forthcoming work [33].

\section{REFERENCES}

[1] Avrami, M., Kinetics of phase change I-III, J. Chem. Phys. 7 (1939), 1103-1112; 8 (1940), 212-224; 9 (1941), 177-184.

[2] Barles, G, Soner, H.M., and Souganidis, P.E., Front propagation and phase-field theory, SIAM J. Cont. Optim. 31 (1993), 439-469. MR1205984 (94c:35005)

[3] Burger, M., Growth and impingement in polymer melts, in: Colli, P., et. al., eds., FreeBoundary Problems, Birkhäuser, Basel, 2003, pp. 65-74. MR2044564

[4] Burger, M., Growth of multiple crystals in polymer melts, European J. Appl. Math. 15 (2004), 347-363. MR2092918 (2006c:80003)

[5] Burger, M., and Capasso, V., Mathematical modelling and simulation of non-isothermal crystallization of polymers, Math. Models and Meth. in Appl. Sciences 11 (2001), 1029-1054. MR.1850561 (2002i:82102)

[6] Burger, M., Capasso, V., and Eder, G., Modelling crystallization of polymers in temperature fields, ZAMM 82 (2002), 51-63. MR1878482 (2003f:82106)

[7] Burger, M., Capasso, V., and Salani, C., Modelling multi-dimensional crystallization of polymers in interaction with heat transfer, Nonlinear Analysis B, Real World Applications 3 (2002), 139-160. MR1941953 (2004b:82056)

[8] Caffarelli, L.A., and Evans, L.C., Continuity of the temperature in the two-phase Stefan problem, Arch. Rational Mech. Anal. 81 (1983), no. 3, 199-220. MR683353 (84g:35070)

[9] Capasso, V., and Salani, C., Stochastic birth-and-growth processes modelling crystallization of polymers in a spatially heterogenous temperature field, Nonlinear Analysis, Real World Applications 1 (2000), 485-498. MR1796584 (2001h:82068)

[10] Chen, G.-Q., and Su, B., Discontinuous solutions in $L^{\infty}$ for Hamilton-Jacobi equations, Chinese Ann. Math. Ser. B 21 (2000), no. 2, 165-186. MR1763491(2001c:35052)

[11] Chen, G.-Q., and Su, B., On global discontinuous solutions of Hamilton-Jacobi equations, C. R. Math. Acad. Sci. Paris 334 (2002), no. 2, 113-118. MR1885091 (2003b:35031)

[12] Chen, G.-Q., and Su, B., Discontinuous solutions for Hamilton-Jacobi equations: uniqueness and regularity, Discrete Contin. Dyn. Syst. 9 (2003), no. 1, 167-192. MR1951317 (2003k:49062)

[13] Crandall, M.G., and Lions, P.L., Viscosity solutions of Hamilton-Jacobi equations, Trans. Amer. Math. Soc. 277 (1983), 1-42. MR690039 (85g:35029)

[14] DiBenedetto, E., Continuity of weak solutions to certain singular parabolic equations, Ann. Mat. Pura Appl. (4) 130 (1982), 131-176. MR663969 (83k:35045)

[15] Eder, G., Crystallization kinetic equations incorporating surface and bulk nucleation, ZAMM 76 (1996), S4, 489-492.

[16] Eder, G., Fundamentals of structure formation in crystallizing polymers, in K. Hatada, T. Kitayama, O. Vogl, eds., Macromolecular design of polymeric materials, M. Dekker, New York, 1997, pp. 761-782.

[17] Evans, V.R., The laws of expanding circles and spheres in relations to the lateral growth rate of surface films and the grain-size of metals, Trans. Faraday Soc. 41 (1945), 365-374.

[18] Evans, L.C., and Gariepy, R.F., Measure theory and fine properties of functions, Studies in Advanced Mathematics, CRC Press, Boca Raton, FL, 1992. MR.1158660 (93f:28001)

[19] Friedman, A., Variational principles and free-boundary problems, Wiley and Sons, Inc., New York, 1982. MR679313(84e:35153)

[20] Friedman, A., and Velazquez, J.L., A free-boundary problem associated to crystallization of polymers, Indiana Univ. Math. Journal 50 (2001), 1609-1650. MR1889073 (2003g:35229)

[21] Kolmogorov, A.N., Statistical theory of crystallization of metals, Bull. Acad. Sci. USSR, Math. Ser. 1 (1937), 355-359.

[22] Ladyzhenskaya, O.A., Solonnikov, V.A., and Ural'ceva, N.N., Linear and quasilinear equations of parabolic type. Translations of Mathematical Monographs, Vol. 23, American Mathematical Society, Providence, R.I., 1967. MR0241822 (39:3159b)

[23] Lions, P.L., Generalized solutions of Hamilton-Jacobi equations, Pitman, Boston, London, Melbourne, 1982. MR667669 (84a:49038) 
[24] Meirmanov, A.M., The Stefan problem, De Gruyter, Berlin, 1992. MR1154310 (92m:35282)

[25] Micheletti, A., and Burger, M., Stochastic and deterministic simulation of nonisothermal crystallization of polymers, J.Math.Chem. 30 (2001), 169-193. MR1921620

[26] Micheletti, A., and Capasso, V., The stochastic geometry of polymer crystallization processes, Stoch. Anal. Appl. 15 (1997), 355-373. MR1454093 (98b:60178)

[27] Monasse, B., and Haudin, J.M., Thermal dependence of nucleation and growth rate in polypropylene by non-isothermal calorimetry, Colloid \& Polymer Sci. 264 (1986), 117-122.

[28] Osher, S., and Sethian, J.A., Fronts propagating with curvature-dependent speed: algorithms based on Hamilton-Jacobi formulations, J. Comp. Phys. 79 (1988), 12-49. MR965860 (89h:80012)

[29] Ratajski, E., and Janeschitz-Kriegl, H., How to determine high growth speeds in polymer crystallization, Colloid Polym. Sci. 274, (1996), 938-951.

[30] Sacks, P.E., Continuity of solutions of a singular parabolic equation, Nonlinear Anal. 7 (1983), no. 4, 387-409. MR696738 (84d:35081)

[31] Schulze, G.E.W., and Naujeck, T.R., A growing 2D spherulite and calculus of variations, Colloid \& Polymer Science 269 (1991), 689-703.

[32] Su, B., and Burger, M., Weak solutions of a polymer crystal growth model, submitted. See also UCLA CAM Report 06-40, July 2006.

[33] Su, B., and Trivedi, R., in preparation.

[34] Taylor, J.E., Cahn, J.W., and Handwerker, C.A., Geometric models of crystal growth, Acta metall. mater. 40 (1992), 1443-1472.

[35] Trivedi, R., and Teng, J., in preparation.

[36] Visintin, A., Models of phase transitions, Birkhäuser, Boston, 1996. MR1423808 (98a:80006)

[37] Ziemer, W.P. Interior and boundary continuity of weak solutions of degenerate parabolic equations, Trans. Amer. Math. Soc. 271 (1982), no. 2, 733-748. MR654859 (83e:35074)

Department of Mathematics, Iowa State University, Ames, Iowa 50011

E-mail address: bosu@iastate.edu

Industrial Mathematics Institute, Johannes Kepler University, Altenbergerstr. 69, A 4040 Linz, Austria

E-mail address: martin.burger@jku.at 